\section{Reputación corporativa: Trustmark y activo de comporta- mientos adquisitivos futuros}

\author{
María Isabel Muñoz Antonin ${ }^{(*)}$
}

Resumen: El presente artículo aborda la temática de la reputación corporativa, relacionándola al valor de marca y vinculándola a la gestión del conjunto de activos y pasivos, sobre una plataforma comunicacional. Para esto, reflexiona en torno al proceso de planificación, mantención y control del activo reputacional, en los que tienen injerencia -entre otros- los esfuerzos de las Relaciones Públicas, analizando a la vez la sensibilidad del relacionamiento de la organización con los públicos estratégicos. Para esto, aborda las implicancias del riesgo reputacional y la concepción de las organizaciones de transformación, en una vigilancia permanente de los recursos intangibles sobre plataformas de comunicaciones y de gestión, para lograr que de verdad sus logos sean capaces de contar una historia corporativa, con pertinencia a objetivos estratégicos e integridad para su credibilidad.

Palabras claves: Comunicación Corporativa - Comunicación Empresaria - comunicación estratégica - Comunicación Institucional - comunicación organizacional - Dircom - Imagen Corporativa - marcas - públicos - Relaciones Institucionales - Relaciones Públicas - Reputación corporativa - stakeholders.

[Resúmenes en inglés y portugués y currículum en las páginas 39-40]

En una época de legítimos cuestionamientos sobre las prácticas y abusos a nivel de gobiernos corporativos, los países y los más complejos mercados hoy se enfrentan a la urgencia mundial para reestablecer equilibrios financieros al amparo de estructuras político-sociales cada vez más eficaces, eficientes, transparentes y sostenibles. Un desafío en el que será determinante el perfeccionamiento de nuevos marcos regulatorios pero también la definición concensuada de un marco valórico que pueda ser exigible a los nuevos sistemas de gestión para la alta gerencia o gobernanza de cualquier actividad productiva del futuro.

El desarrollo de la nueva economía demandará prioridades no sólo focalizadas en la mera acumulación de capital, el libre y fluido intercambio o la estabilidad de las monedas, también la consolidación de buenas instituciones a nivel societal (Sorman, 2008), en un lento proceso de tránsito hacia la recuperación de las confianzas.

Tal como lo grafica la escuela de pensamiento clásica sobre autopoiesis de Maturana, más algunas demostraciones empíricas dadas por casos ejemplificadores de grandes empresas, se puede afirmar que todas las organizaciones requieren igualmente la ocurrencia de equilibrios vitales que aseguren su funcionamiento e higiene, como clave de vitalidad para la permanencia.

No parece extraño entonces, que más allá de los siete hábitos esperables en personas altamente 
efectivas, hoy ya se hable con propiedad del octavo hábito de las organizaciones (Covey, 2008) expresado en el valor de la confianza, como una virtud social, motor económico y competencia flexible, que se pudiera traducir en la capacidad para incrementar o no la rentabilidad, para sobrevivir o no a la hipercompetencia, para justificar o no su fin o declaración de propósitos, en términos de su más intrínseca operatividad y eficiencia en el uso de recursos.

La hegemónica eclosión de Internet como parte de esta Nueva Economía y la proliferación de transacciones globales B2B desataron también el replanteamiento de antiguas formas de interacción y cooperación entre empresas u organizaciones, desafiando a la adopción de modos distintos de relación lo que implica de ahora en adelante una reorganización profunda en el funcionamiento de los negocios, muchos de los cuales han decidido operar en red como forma organizativa y a una transformación socio-técnica que ha afectado en consecuencia tanto procesos de creación, intercambio y distribución de valor de cada una de ellas (Castells, 2001, p. 83). Esta geometría variable, propia de la estrategia empresarial aprendida por la nueva empresared, aprovecha los nuevos medios tecnológicos que otorga la gran nube, para potenciar su escalabilidad, interactividad, flexibilidad, producción personalizada (a medida del consumidor), sumado al complejo desafío, en algunos casos, de una gestión de marca global.

Este sinérgico desgaste diario de la nueva empresa-organización, implicará por tanto un esfuerzo amplio y multilateral, mayores controles e inversión-inyección de innovación, férreo control de la calidad final del producto, retroalimentación positiva de todos los agentes interactuantes en el proceso de producción (ex ante y ex post), venta y post-venta, como la oportuna detección y corrección de anomalías, para evitar correr el riesgo de perder o dañar el frágil indicador de reputación.

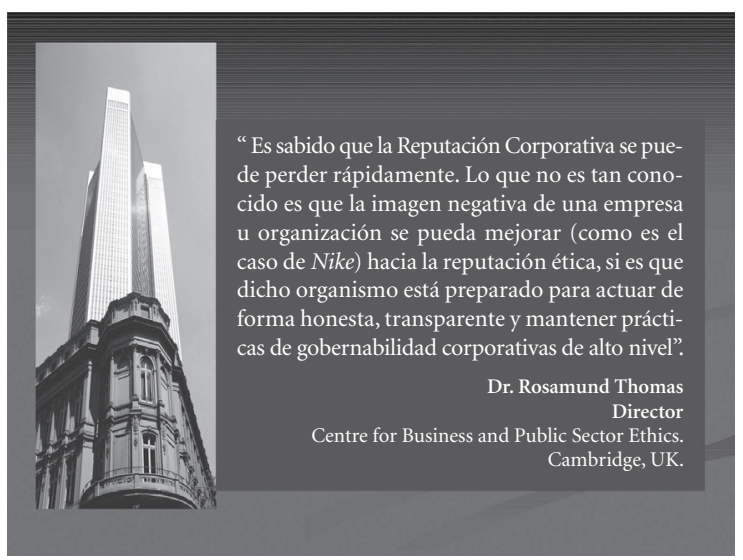

Figura 1: La Reputación Corporativa.

Fuente: Ponencia María Isabel Muñoz en Academia de Guerra. Ejército de Chile (2009).

\section{Una espuma que sube y baja}

La reputación, entendida basalmente como el activo resultado de la sumatoria de percepciones pasadas, presentes, y expectativas futuras de grupos de interés multisectoriales respecto de 
una compañía u organización (público o privada), es también el resultado de una evaluación ponderada de calidad y nivel de desempeño por parte de estos mismos agentes de influencia en una línea determinada de tiempo. Lo anterior, sobre la base de indicadores medibles y por tanto gestionables. Bien lo resumió un artículo publicado el 2003 por The Financial Times, donde Alison Maitland, expresó: "Uno sólo se da cuenta de lo que vale cuando está hecha pedazos". Así, tanto la reputación como la imagen de una organización provienen de la conciencia y valor percibido, si consideramos que cualquier comportamiento adquisitivo estará subyugado a un conjunto de circunstancias, valores y lealtades de grupos cada vez más definidos.

En este sentido, hablamos de un proceso esencialmente dinámico, donde no sólo las acciones o no acciones de una estructura privada o pública afectarán la reputación, sino también lo que sucede en su entorno y lo que hace o no su competencia directa, coyuntura que podría afectar al proceso de manera importante.

Para enfrentar esta incertidumbre, algunos expertos (Fernández, 2005) recomiendan la ejecución de un monitoreo constante para identificar las oscilaciones o varianzas significativas en segmentos socio-mercadotécnicos, a fin de poder determinar a tiempo acciones efectivas y correctivas, tanto internas y externas, para influir positivamente en el esquivo delta de percepción y valorización. Aquello obliga entonces a considerar en el análisis y planificación, cuestionamientos claves:

1. ¿Quiénes son los clientes (usuarios/ciudadanos) y cómo muestrear correctamente?

2. ¿Cuáles son los meta-canales de comunicación de los actores principales?

3. ¿Cuál es el status actual de reputación de la organización dentro de su sector?

Estas preguntas son fundamentales a objeto de evitar incurrir en los seis grandes errores, sintomáticos de una mala gestión de riesgo reputacional, como:

a) confiar en datos históricos;

b) centrarse en indicadores limitados;

c) pasar por alto riesgos conocibles;

d) subestimar riesgos ocultos;

f) fracasar en la comunicación y,

g) no negociar (reaccionar) en tiempo real.

\section{Reputación y equidad de marca}

La Reputación, ya antes definida, también cruza de manera crítica la equidad de marca, si se tiene en cuenta que a partir de su gestión dependerán un conjunto de activos y pasivos de ella misma. El sello reputacional permanece estrechamente unido al nombre de la marca y al logo, y en este sentido la reputación puede dar o quitar al valor dado por un producto o servicio, generando retornos tanto a los consumidores como a la firma. En consecuencia, el proceso de planificación, mantención y control del activo reputacional debe contemplar ineludiblemente la consideración de los cinco activos más importantes la equidad de marca, a saber:

1. Lealtad de marca

2. Conciencia del nombre de la marca 
3. Calidad percibida de la marca

4. Asociación de la marca sumada a la calidad percibida

5. Otros activos de propiedad de la marca

Mirado el pentágono del Brand Equity, sea quizás la asociación de marca o imagen percibida uno de los aspectos más cruciales de la equidad. Junto a ella, la calidad percibida como otro activo distinto de la asociación de marca. Ésta se ha transformado en un fondo de negocios muy importante para muchas firmas y la motivación de programas diseñados para mejorar la equidad de marca.

Conclusiones arrojadas por importantes investigaciones, entre ellas las del Instituto de Planificación Estratégica (PIMS) con análisis empíricos de información financiera y operacional de más de 3.000 compañías, han mostrado que la calidad percibida es el único y más importante factor de retorno de la inversión medido por la entidad norteamericana (The Strategic Planning Institute, $\mathrm{s} / \mathrm{f}$ ).

En paralelo, un estudio realizado a 250 gerentes -quienes identificaron las ventajas competitivas sostenibles de su firma- fue precisamente la calidad percibida identificada como la variable de mayor ponderación. Se aborda así una consideración estratégica suficientemente importante y aceptada como para ser incluida como una dimensión importante de la reputación.

Una adecuada planificación puede impactar positivamente una curva. Así lo demostró el importante repunte obtenido por la tabacalera Chiletabacos, de acuerdo al último estudio (2008) elaborado por Reputation Institute y la Universidad Católica de Chile, que identifica a "Las empresas con mejor reputación en Chile", donde la filial chilena de B.A.T., tras un castigado periodo en el cual tuvo que enfrentar una despiadada tempestad publicitaria al amparo de la nueva Ley del Tabaco el 2006, repuntó de 7.1 puntos el 2007 a 11.03 puntos el 2008. Esta cifra no sólo dio cuenta de un cambio progresivo en las percepciones y los hábitos de consumo a nivel social, sino también evidenció un asumido cambio de conducta propiciado por la propia empresa -que para legitimar su negocio y hacerlo sostenible, a fin de no oponerse a las fuerzas del entorno-, optó por armonizar aceleradamente la relación entre fumadores y no fumadores propiciando un nuevo paradigma del consumo responsable.

\section{Oscilaciones de la reputación}

Aunque para algunos consultores los principales pivotes considerados sean factores como la gestión, productos, servicios, actividades, trabajadores y organización, lo importante es no perder de vista que la mecánica y oscilaciones cíclicas de este "preciado factor" no sólo impacta ámbitos privados o propios del sector productivo. Un buen ejemplo de ello y dado por los resultados de este mismo estudio en Chile, posiciona el sistema de transporte público Transantiago históricamente como la imagen-marca peor evaluada en todas las mediciones realizadas por Reputation Institute a la fecha. Es decir, nunca antes un proyecto o iniciativa gubernamental había bajado al puntaje 5.2, constituyéndose a nivel internacional como hito estadístico y "caso de análisis" aunque lamentablemente poco feliz para una parte importante de los ciudadanos chilenos.

Es sin más, la ponderación más baja junto a la calificación obtenida por la Empresa de Ferrocarriles del Estado-EFE (30.31), a partir de un universo de 2.400 personas encuestadas, lo que denota una enorme y compartida desconfianza, que ha castigado la proliferación de múltiples 
y sucesivos fracasos a nivel de la administración pública, con un millonario déficit financiero que como agravante involucra dineros públicos y una gestión administrativa percibida como deficiente, a juicio de sus propios stakeholders.

Conclusiones igualmente decidoras se desprenden de la evolución de indicadores de reputación catastrada por otro estudio, en función del performance histórico y diferencias estadísticamente significativas entre empresas e instituciones, donde marcas como Coca-Cola, Nestlé y Soprole demuestran que este activo es gestionable, pero que por otra parte no da tregua y tampoco ofrece garantías de continuidad a menos que se corrijan a tiempo las desviaciones generadas a nivel estructural, en cualquiera de los ejes que sostienen la reputación.

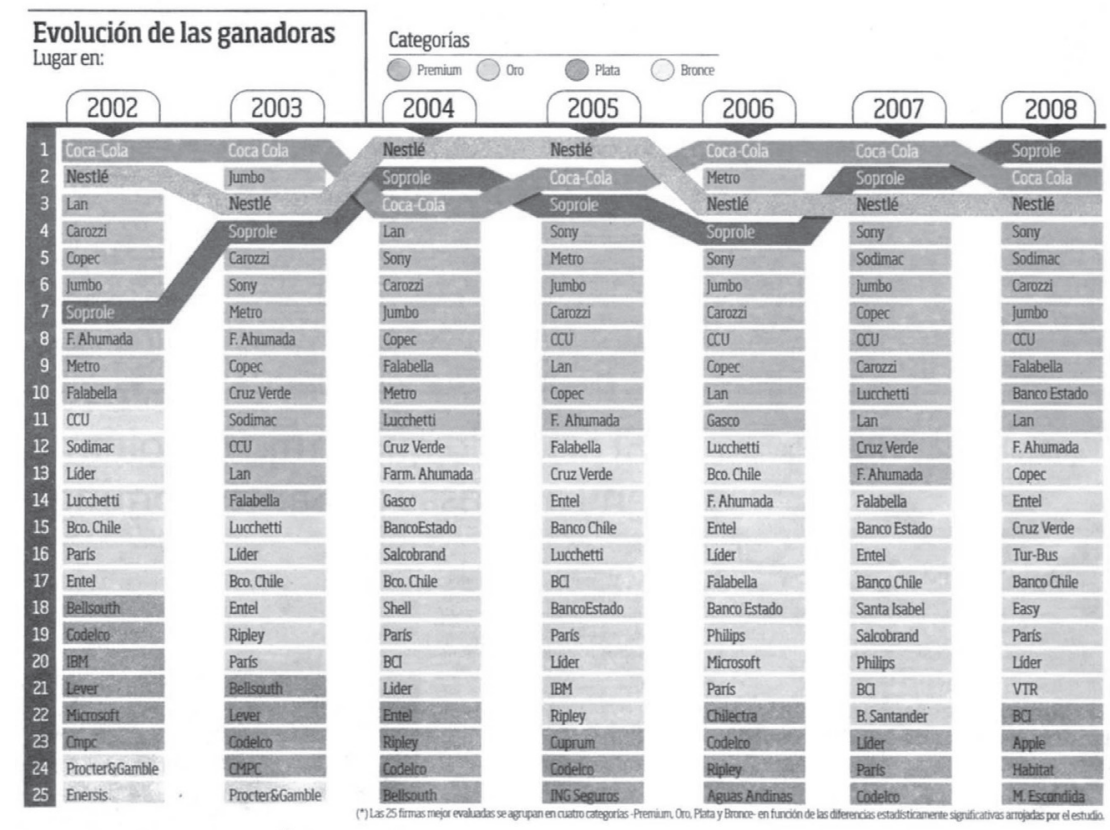

Figura 2: Ranking de Reputación Corporativa Hill \& Knowlton Captiva.

Fuente: La Tercera (diciembre 14, 2008).

En el caso de este estudio, se trata de una herramienta desarrollada por la agencia Hill \& Knowlton Captiva, diario La Tercera y Collect-GFK, para medir la Reputación Corporativa a nivel de consumidores. Éste se basa en la extrapolación de datos a partir de una batería de preguntas que versa sobre las seis dimensiones que sustentan la reputación, ergo: Relación emocional, desempeño financiero, ambiente laboral, responsabilidad social, administración y productos; y servicios; levantando indicadores (escala 0-1000) que permiten establecer las primeras 25 empresas u organizaciones, a partir de la información resultante de unas 4.300 encuestas telefónicas aleatorias aplicadas en hogares de Santiago de Chile. 
Integrando el primer quintil y por séptima vez, se consolidaron el 2008 en la primera ubicación la marca de productos lácteos Soprole, seguida de cerca por Coca-Cola y Nestlé, como líderes en Chile en el ranking de Reputación Corporativa, de acuerdo a este importante sondeo de opinión.

LA TRRCERA Domingo 14 de didiembre de 2008

Ranking de Reputación Corporativa 2008

\begin{tabular}{|c|c|c|c|c|c|c|c|c|c|c|c|c|}
\hline \multicolumn{13}{|c|}{$\begin{array}{l}\text { Fortalezas y debilidades de las ganadoras } \\
\text { Clasificadón según las seis dimensiones que componen la reputación. }\end{array}$} \\
\hline Emocional & & $\begin{array}{l}\text { Desempeñ } \\
\text { financiero }\end{array}$ & & $\begin{array}{l}\text { Responsabili } \\
\text { social }\end{array}$ & & \multicolumn{2}{|c|}{$\begin{array}{c}\text { Ambiente } \\
\text { laboral }\end{array}$} & \multicolumn{2}{|c|}{$\begin{array}{l}\text { Gestióny } \\
\text { liderazgo }\end{array}$} & \multicolumn{2}{|c|}{$\begin{array}{l}\text { Productosy } \\
\text { servicios }\end{array}$} & \multirow{5}{*}{$\begin{array}{l}\text { entran al ranking 2008: } \\
\text { Tur Bus, Easy, VTR, } \\
\text { Apple, Habitat y } \\
\text { Escondida. Ninguna } \\
\text { había clasificado antes. }\end{array}$} \\
\hline Sodinax EI & & acsa & & gorale & 806 & Sopole & & & & rele & 0855 & \\
\hline Sunde -80 & & Falabolla & 822 & Banco Estado & 793 & mercala & 84 & Sony & 1060 & Sony & 802 & \\
\hline Sony $\quad x$ & & Lanchle & 80 & Nestik & 70 & Sany & 811 & Sopride & 61 & Nestle & 85 & \\
\hline Nester & & Liter & 816 & Sodinac & $\pi 9$ & Sodinac & 803 & Sodinx & 850 & Sodinax & BS & \\
\hline Canesi & & Junbo & 800 & Canozi & $\pi 9$ & Netile & 2001 & liester & 840 & $\operatorname{coc} \cos ^{2}$ & 800 & \\
\hline $\operatorname{cosc} \cos ^{2} \pi$ & & Nestle & 79 & $\infty$ & $\pi 4$ & Caroui & 798 & Falbible & 899 & Jumbo & 839 & \\
\hline Bano Estado $\pi$ & & Parts & & junbo & & w & 799 & Lan & 837 & Carrexi & 806 & \\
\hline Falabele $\pi$ & & F.Ahumada & $\pi 9$ & Sorny & 20 & Junto & $\pi 2$ & Junbo & $\approx 5$ & cu & 916 & \\
\hline copec $\pi$ & & Sodinax & $7 m$ & 5000 & 765 & Bannotsado & $m$ & cal & 89 & LanCule & 908 & \\
\hline Jumbo $x$ & & Coper & 58 & Falabela & 735 & Fabbella & $\pi 0$ & Caroeni & 831 & Fabbella & 804 & empresas salen delas top \\
\hline cou $\quad x$ & & Sony & $m s$ & Banochile & 78 & Lan & 78 & Tur-Bus & 800 & Banno Etatio & $\infty$ & 25 este año: Lucchetti. \\
\hline EAhurade $x$ & & cou & $\pi 1$ & Eary & $\pi 4$ & Bancochile & 743 & Entel & 705 & F. Ahumada & 79 & Salcobrand, Santa Isabel, \\
\hline Tur-Bus $n$ & 73 & Carromi & 749 & F. Alumida & $n 0$ & F. Ahumadi & 74 & copec & 785 & Gneverde & $\pi$ & Santander,Philipsy \\
\hline Entel $\pi$ & 706 & Sopale & 742 & Cruz Verde & $m 7$ & Cnuvende & 30 & BancoEstado & 794 & Enter & $\pi 8$ & Codelco. \\
\hline Creverente $x$ & $m$ & Entel & 73 & Lan & 73 & Etat & $7 s$ & E. Ahumadi & 791 & Coper & $\pi 6$ & \\
\hline Easy & $\pi 16$ & Bano Estado & $7 \pi$ & Entel & $\pi 5$ & Easy & $\pi \theta$ & Cunglerde & $m$ & Easy & $\pi 5$ & \\
\hline Lan & $\pi 1$ & Exy & 728 & Tur-Bis & 703 & Copec & $\pi B$ & Lider & $m$ & Tur-Bus & nos & \\
\hline Paris & mo & Tur-Bls & $\pi 0$ & Coper & 6 & Ba & 72 & Bancochile & $m 0$ & Paris & 79 & \\
\hline Bancoutive $\pi$ & mos & Bannochile & $\pi 7$ & $\mathrm{BC}$ & 64 & Turbus & no & Parls & $\pi 66$ & Bannolite & 748 & \\
\hline VIR & 695 & VIR & $\pi$ & VIR & 608 & Paris & 304 & Easy & 700 & Apple & $\pi 06$ & \\
\hline Liter & $\theta$ & Conallente & 78 & Apple & $\infty$ & Apple & ter & VIR & 702 & $B O$ & 74 & acionales figuran \\
\hline $\mathrm{BO}$ & si9 & BC & $\pi 3$ & Paris & (5) & VIR & $\infty$ & BD & $n$ & VTR & TR & $\begin{array}{l}\text { en elranking, solouna } \\
\text { estatal y las } 17\end{array}$ \\
\hline Habitat a & 612 & MEscondida & $6 / 3$ & Lifer & 650 & Loer & $\varpi$ & Apple & $\pi 8$ & Lider & 75 & $\begin{array}{l}\text { estataly las If } \\
\text { restantes son de grupos }\end{array}$ \\
\hline Aple & $6 \infty$ & Apple & 69 & Habitat & 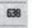 & MEscminith & 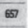 & Hadite & 84 & Hatilat & 61 & restantes son de grupos \\
\hline MEscondict 5 & 80 & Habiet & 650 & MEsundida & $\varpi$ & Hatita & GR & MEsconditita & $\infty$ & MEssantica & $\omega$ & \\
\hline
\end{tabular}

Figura 3: Ranking de Reputación Corporativa.

Fuente: La Tercera (diciembre14, 2008)

En el comportamiento promedio de la galería de empresas y multinacionales que lideran esta clasificación, se aprecia una fluctuación dinámica pero al mismo tiempo disímil para cada una de las seis dimensiones (cada una en estrecha relación con legítimas prioridades y necesidades intrínsecas de sus cadenas de valor), a lo que se agrega un delta generado por la inestabilidad de indicadores financieros, la incertidumbre propia de escenarios económicos vinculados; modelos de gestión acoplados a cadenas productivas de alta complejidad y estilos de liderazgo corporativo distintivos, lo que a muchas de estas compañías les ha significado subir algunos ítems pero también bajar en otros.

A fines del 2009 Coca-Cola recupera su habitual liderazgo demostrando la compañía una sólida consistencia corporativa, ejemplar a nivel promedio en todos los ejes. También sube dos posiciones la marca Sony como correlato de lo "amigable" que se ha perfilado la marca y la alta valorización ciudadana respecto de su percepción en servicios ligados a la industria de tecnología, wellness y entretención Ello explicaría que figuren el 2009 dentro de los primeros 
20 lugares marcas que no habían estado nunca antes presentes en estos tramos desde el 2002 al 2008 , como Adidas $\left(5^{\circ}\right)$, Nike $\left(6^{\circ}\right)$, Samsung $\left(15^{\circ}\right)$, Clínica Alemana $\left(17^{\circ}\right)$ y LG $\left(19^{\circ}\right)$.

\section{Ranking general 2009}

\begin{tabular}{|c|c|c|c|c|c|c|}
\hline \multirow{2}{*}{ Ranking Oro $=$} & \multicolumn{2}{|c|}{ Plata } & \multirow{2}{*}{\multicolumn{2}{|c|}{2008}} & \multirow{2}{*}{\multicolumn{2}{|c|}{2009}} \\
\hline & & 2007 & & & & \\
\hline 1 Coca Cola & 루 & 813 & 는 & 824 & 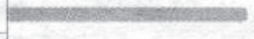 & 826 \\
\hline 2 Sony & 루 & 805 & - & 822 & 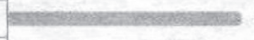 & 820 \\
\hline 3 Nestlé & e & 807 & ㄴ. & 823 & 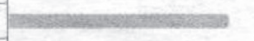 & 807 \\
\hline 4 Soprole & - & 809 & $\theta$ & 833 & 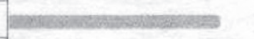 & 798 \\
\hline 5 Adidas & ce & & (2) & - & 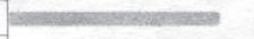 & 798 \\
\hline 6 Nike & 은 & - & e & - & 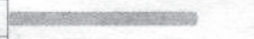 & 775 \\
\hline $7 \mathrm{CCU}$ & - & $7 \pi$ & 를 & 793 & 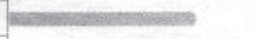 & 773 \\
\hline 8 Metro & - & - & $\theta$ & . & 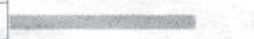 & 773 \\
\hline 9 Lucchetti & - & 760 & $\theta$ & 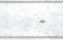 & 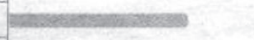 & 765 \\
\hline 10 Junbo & 룽 & 790 & - & 802 & 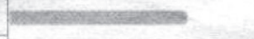 & 764 \\
\hline 11 Lab. Chile & ;) & $\cdots$ & 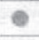 & - & 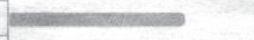 & 762 \\
\hline 12 Sodimac & - & 790 & 6 & 819 & 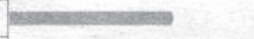 & 750 \\
\hline 13 Carozzi & - & 775 & 당 & 809 & 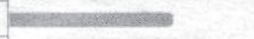 & 750 \\
\hline 14 Copec & 둥 & 782 & 둥 & 752 & 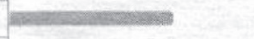 & 750 \\
\hline 15 Samsumg & 을 & & 눙 & - & 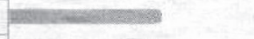 & 738 \\
\hline 16 LAN & 둥 & 766 & 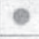 & 780 & 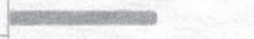 & 734 \\
\hline 17. Clínica Alemana & - & . & 른 & $\cdot$ & 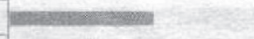 & 730 \\
\hline 18 Entel & - & 739 & - & 752 & 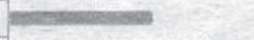 & 729 \\
\hline $19 \mathrm{LG}$ & e & 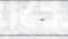 & - & - & 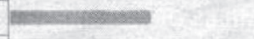 & 727 \\
\hline 20 Banco Estado & - & 742 & 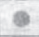 & 788 & 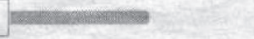 & 725 \\
\hline
\end{tabular}

Figura 4: Ranking de Reputación Corporativa Hill \& Knowlton Captiva. Collect GFK. Fuente: La Tercera (octubre 28, 2009).

La escandalosa evidencia en Chile de una eventual política de colusión a nivel de precios que afectó el 2009 a las cadenas de Farmacias Ahumada, Cruz Verde y Salcobrand, las sacó abruptamente de la lista, reposicionando otra marca ligada al rubro, Laboratorio Chile $\left(11^{\circ}\right)$, que se perfiló como marca alternativa para el suministro general de medicamentos en plena emergencia de la crisis.

Quedó en evidencia que todos los progresivos esfuerzos de Relaciones Públicas (PR), publicitarios e inversiones de marketing que permitió a estas marcas ocupar un sitial y acumular cierto stock reputacional hasta el año 2008, fueron insuficientes descuidando lo más sensible de sus públicos estratégicos: ¡la confianza en su promesa de valor!. A pesar que el consumo, por un tema de disponibilidad de oferta versus demanda, no se haya mermado significativamente, el detrimento de este activo fue crítico y seguramente tomará mucho tiempo revertir el daño y la percepción general a nivel del mercado de consumidores chilenos. 


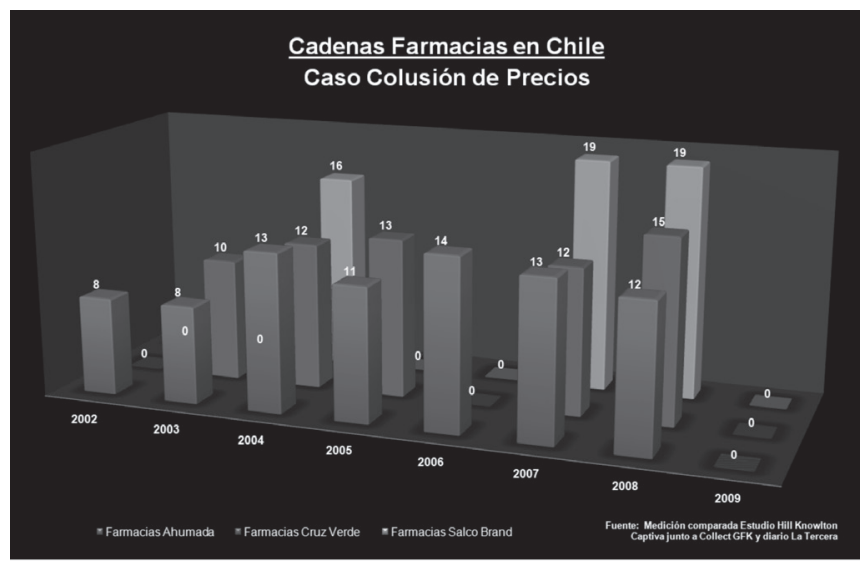

Figura 5: Cadenas de Farmacias en Chile.

Fuente: Escuela de Relaciones Públicas Corporativas de UNIACC sobre la base de datos comparados del estudio Hill \& Knowlton Captiva. Collect GFK y La Tercera.

\section{¿Cómo una organización maneja sus malas noticias?}

Este aspecto puede convertirse en el elemento más importante, como indicador de lo que es o no capaz de hacer una compañía para determinar su reputación corporativa global. Es precisamente en tiempo de crisis cuando las "trustmarks" se forjan, se consolidan o se pierden. (Darrell Bricker, 2007). Gestionar de manera eficaz el riesgo reputacional, involucra necesariamente cinco pasos:

a) Evaluar la reputación de su empresa a nivel multistakeholders: medición de la reputación en múltiples áreas, de forma contextual, objetiva y cuantitativa, para contestar preguntas como: ¿cuál es la reputación de la empresa en cada área? ¿Por qué? ¿Cómo se comparan estas reputaciones con las de otras empresas u organizaciones del sector?

b) Evaluar el verdadero carácter de la empresa: por sobre la tendencia gerencial a sobreestimar sus propias capacidades o suponer que se posee una buena reputación si no hay indicios que ésta sea mala, cuanto más contextual, objetivo y cuantitativo sea este sondeo será mejor (uso de herramientas como el Extensible Business Reporting Language (XBRL), que permite que cada pieza de información de un estado financiero sea etiquetada electrónicamente para ser importado hacia un software analítico en forma rápida y con bajos costos; software de visualización, etc.)

c) Cerrar las brechas entre reputación y realidad: es aconsejable disminuir las brechas a través de un programa eficaz de comunicaciones y relaciones con grupos y agentes de influencia. $\mathrm{Si}$ una reputación es injustificadamente positiva, la organización está obligada a mejorar sus capacidades, conducta y desempeño, para moderar las percepciones del entorno. 
d) Monitorear creencias y expectativas cambiantes: el desarrollo de encuestas o programas de sondeo regulares, pueden revelar cambios u oscilaciones abruptas a nivel de expectativas.

e) Considerar el reclutamiento de un alto ejecutivo: cuya posición sea de rango inferior al Chief Executive Officer (CEO), dotado de las competencias y habilidades para liderar esta área estratégica.

\section{¿Cuánto vale mi marca?}

La multinacional Interbrand, conocida como una entidad especializada en el proceso de valorización de marcas, las clasifica multiplicando el beneficio diferencial de ésta por un múltiplo. No se abordará aquí el desarrollo metodológico matemático para el cálculo de este índice diferencial, pero sí se considera relevante destacar que aquel múltiplo se determina cuantificando factores claves que, según este modelo, determinan en si la "fortaleza de la marca", dados por la ponderación de los siguientes siete ítems:

\begin{tabular}{|l|l|}
\hline Liderazgo & $\begin{array}{l}\text { Una marca líder es más estable y tiene más valor que otra marca con una cuo- } \\
\text { ta de mercado menor porque el liderazgo proporciona influencia en el mercado, } \\
\text { para poder establecer precios, dominio en la distribución, mayor resistencia } \\
\text { a los competidores, etc. }\end{array}$ \\
\hline Estabilidad & $\begin{array}{l}\text { Obtienen altas puntuaciones en este factor las marcas establecidas y que } \\
\text { cuentan con la lealtad de los consumidores. }\end{array}$ \\
\hline Mercado & $\begin{array}{l}\text { Una marca en un mercado estable y creciente con elevadas barreras de entra- } \\
\text { da, puntuará muy alto. }\end{array}$ \\
\hline Internacionalidad & $\begin{array}{l}\text { Marcas que operan en mercados internacionales tienen más valor que mar- } \\
\text { cas nacionales o regionales. Todas las marcas no son capaces de atravesar las } \\
\text { barreras culturales y nacionales. }\end{array}$ \\
\hline $\begin{array}{l}\text { Trayectoria de } \\
\text { la marca }\end{array}$ & $\begin{array}{l}\text { La tendencia de una marca a mantenerse actualizada y relevante para el } \\
\text { consumidor incrementa significativamente su valor. }\end{array}$ \\
\hline Apoyo & $\begin{array}{l}\text { Marcas que han recibido inversiones y apoyo deben considerarse más valio- } \\
\text { sas que aquellas que no lo han recibido. }\end{array}$ \\
\hline Protección legal & $\begin{array}{l}\text { La robustez y amplitud de la protección de marca (monopolio legal) puede } \\
\text { ser un factor crítico en su valorización. }\end{array}$ \\
\hline
\end{tabular}

Figura 6: Modelo de valorización Interbrand para "fortaleza de marca".

Fuente: http://www.interbrand.com 
Otro método de valorización, utilizado por la empresa Houlihan Valuation Advisors, considera entre sus variables de rentabilidad un importante porcentaje de activos intangibles para medir la progresión cuantitativa y cualitativa de sus principales brand value drivers.

\begin{tabular}{|l|l|l|l|l|l|}
\hline Variables & $\begin{array}{l}\text { Rentabilidad } \\
\text { exigida }\end{array}$ & $\mathbf{2 0 0 7}$ & $\mathbf{2 0 0 8}$ & $\mathbf{2 0 0 9}$ & $\mathbf{2 0 1 0}$ \\
\hline Circulante neto & $6 \%$ & $\rightarrow$ & $\rightarrow$ & $\rightarrow$ & $\rightarrow$ \\
\hline Inmovilizado & $9 \%$ & $\rightarrow$ & $\rightarrow$ & $\rightarrow$ & $\rightarrow$ \\
\hline Activos intangibles & $14 \%$ & $\rightarrow$ & $\rightarrow$ & $\rightarrow$ & $\rightarrow$ \\
\hline Patentes & $15 \%$ & $\rightarrow$ & $\rightarrow$ & $\rightarrow$ & $\rightarrow$ \\
\hline Tecnología propia & $20 \%$ & $\rightarrow$ & $\rightarrow$ & $\rightarrow$ & $\rightarrow$ \\
\hline Free cash flow de la empresa & & & & \\
\hline - activos utilizados x rentabilidad exigida & \multicolumn{5}{l}{ = Valor actual (Free cash flow). } \\
\hline Free cash flow atribuido a la marca & Porcentaje crecimiento. \\
\hline Valor de la marca &
\end{tabular}

Figura 7: Reducción modelo original

Fuente: HVD http://www.houlihan.com

Como indica la figura anterior, cualquiera sea la cadena de valor o proceso productivo, el cálculo de valoración de marca aumenta de manera consistente la información que sobre la propia marca tiene una empresa $u$ organización, como herramienta gerencial de apoyo y complemento a una estrategia, coherente a lo largo del tiempo, para asignar de manera racional recursos de marketing, y en definitiva crear valor. Durante la última década la integración de activos de tipo intangibles a la arquitectura de datos e información disponible en sistemas, mapas de conocimientos y sistemas colaborativos de gestión ha sido más bien gradual aunque de crecimiento sostenido. Tal vez lo destacable de este modelo, sea la consideración de un 14\% por sobre otras variables porcentuales como el circulante neto y activos inmovilizados, durante todo el proceso de estimación de valor. Todas ellas convertidas hoy en plataformas y Conditio sine qua non bajo las cuales operan los mercados globalizados, especialmente aquellos que funcionan bajo la modalidad 7x24 en línea, que constituye un sistema de operación siete días a la semana, las 24 horas del día.

En este complejo desafío de generación de valor, las empresas u organizaciones de alto desempeño, no sólo deberán aumentar la velocidad decisional y operativa, sino también conquistar nuevos market places electrónicos, administrar sistemas estratégicos sobre la base de decisiones sincrónicas (más que diacrónicas); consolidar una integración interna y externa, y de paso adquirir capacidad de diseño de sistemas únicos de Client Relationship Management (CRM) para su relación con clientes. 
Sumado a todo lo anterior, y como si esto fuera poco, se requerirá definir estructuras tecnológicas de vanguardia que se adapten con comodidad al modelo de cada negocio, resolver la paradoja de la seguridad digital, externalizar servicios estratégicos y/o desarrollar verdaderas fábricas de procesos adaptativos (Melnick, 2008) a las que muy bien se refiere el modelo Estrategia, Tecnología, Adaptabilidad y Negocios (ETAN). (Melnick y Barraza, 2008).

Bajo este escenario, aquellas marcas que busquen perfilarse como reconocidas 'organizaciones de transformación', requerirán además ingresar decididamente al mundo de los recursos intangibles, permitiendo el acople de efectivas plataformas de comunicaciones y gestión multidimensional a su operación, y más aún, vigilando una administración planificada de activos reputacionales con y para sus más diversos públicos. ¡Vaya desafío!

Será clave para la alta gerencia efectuar exhaustivos mapeos para evitar incurrir en el "error del tercer tipo", es decir, consignar erráticamente todos los esfuerzos para resolver la pregunta equivocada. Aquello demandará de las empresas u organizaciones, desde una dimensión más bien práctica, el desarrollo de una capacidad metanoica que reformule y repiense su propio foco, ángulos y perspectivas para la resolución de problemas y adaptación de nuevos sistemas de gestión, hacia modelos de alto desempeño y valorización dirigida multistakeholders.

Tal es la importancia actual de una gestión óptima en la administración de activos intangibles, que muchas empresas no sólo invierten sumas millonarias para capitalizar futuros dividendos, sino también ha significado el reposicionamiento u emergencia de una nueva generación de profesionales cada vez más especializados en la comunicación, planificación y administración de relaciones.

Un reciente estudio publicado por McKinsey (Quarterly, 2008), acerca del estado actual de la filantropía corporativa, donde participaron 721 ejecutivos de todo el mundo (74\% eran CEO’s), concluye que estas prácticas son hoy consideradas a nivel transversal como una herramienta efectiva para las empresas, pero que éstas no sólo persiguen cumplir con los objetivos sociales declarados, sino obtener beneficios concretos a partir de estos mismos programas y proyectos, para a través de ellos incrementar su indicadores de reputación corporativa e imagen de marca, dicho de otro modo, toda vez que su valor bursátil se incrementa a partir de estas acciones y seudo intenciones.

\section{Ecuación virtuosa}

Con más o menos matices, en el ejercicio descriptivo del concepto reputacional, nos referimos a una simbiosis de la que debe hacerse cargo una organización, sea por la eficacia o inoperancia de su motor organizacional. En ello participarán conceptos y variables estructurales, que sin ser lo mismo, van estrechamente concatenados. Si la reputación dice relación con lecturas y decodificaciones emotivas y multidimensionales, una marca corporativa fuerte y consolidada superará con creces la mera colección de experiencias que pueda poseer una industria o institución (Schulze, 2008), brindando mayor valor a sus actuales o posibles transacciones periféricas, y por otro lado, forjando una fuerte y duradera conexión emocional con los grupos de clientesusuarios (Granoff, 2008); en un proceso en esencia dinámico e inevitablemente impredecible. La sola delimitación de fronteras de cada marca ya es en si una complejidad. En ocasiones, la tentación de utilizar una estrategia de paraguas corporativo único para amparar múltiples marcas, podría incluso atrofiar seriamente el crecimiento de utilidades e ingresos de una em- 
presa. Porque no siempre una marca, aún cuando ésta provenga de un mismo conglomerado corporativo o grupo económico, posee la misma reputación o incluso conexión emotiva con sus grupos de clientes. El posicionamiento aspiracional, por ejemplo, de la marca Estee Lauder es diametralmente distinto de la individualidad irreverente de $M A C$ o de la minimalista simplicidad del sello Clinique.

En consecuencia, confundir ambos aspectos fusionando marcas sin considerar las expectativas y castigos de sus públicos clientes, podría culminar con lapidarios éxodos de consumidoresusuarios y pérdidas millonarias, redundando finalmente en detrimentos irreversibles a nivel de imagen. Contar con poderosos activos como un ADN Corporativo, conexiones con clientes, presencia mediática y posicionamientos de estilos de vidas o promesas distintivas asociadas a la marca, podría demandar igualmente estrategias localizadas, apoyos logísticos específicos, no excluyentes, paralelos o tal vez integrados, donde de verdad sus logos sean capaces de contar una historia corporativa o institucional, con pertinencia a objetivos estratégicos y fundamentalmente con integridad para su credibilidad futura.

Contrario sensu, y sin desconocer las proposiciones más tradicionales formuladas por el marketing, existen algunas compañías que por medio de prácticas, en ocasiones abusivas, increíblemente alientan a sus públicos para que éstos suscriban malas decisiones de compra, dado que sus utilidades dependen de las carteras más insatisfechas o desorientadas. Lamentablemente, éstas han descubierto que en ocasiones masas de clientes confundidos y dramáticamente desinformados a menudo terminan tomando pésimas decisiones, llegando incluso a ser muy rentables a largo plazo (pagos por comisiones y penalizaciones por incumplimiento).

Algunas preguntas claves para tener en cuenta:

- ¿Son sus clientes más rentables aquellos que tienen las mejores razones para estar insatisfechos con su organización?

- ¿Existen reglas que requiere que los clientes rompan porque al hacerlo generan utilidades?

- ¿Procura que a sus clientes les cueste entender o acatar sus reglas, y de hecho, les ayuda a violarlas?

- ¿Depende de contratos o cláusulas para evitar que los clientes se vayan?

Todas ellas interrogantes destinadas a detectar síntomas claros de un 'marketing agresivo' pero no necesariamente sostenible desde el punto de vista de la reputación y rentabilidad a largo plazo. Especialmente en épocas de contracción o recesión económica, los cuestionamientos deberán ser del todo estratégicos y orientados directamente a sus grupos de influencia: ¿qué quieren los accionistas? ¿Qué es lo que quieren los analistas? ¿Qué quieren o necesitan los clientes? ¿Qué buscan y necesitan los empleados? ¿Qué quieren los proveedores, el Gobierno y la Administración Pública? ¿Qué esperan los medios de comunicación y la sociedad? (el entorno más inmediato). Para a partir de este proceso de escucha, poder construir con consistencia el diseño de una estrategia coherente e integrativa. Estados de alta insatisfacción y rotación deberían ser particularmente preocupantes para dichas compañías o instituciones, al comprobar que sus clientes o usuarios migran hacia su competencia, que de seguro ofrecerá al asecho una alternativa mucho más amigable y por cierto transparente, denotando que no hay terreno de cultivo para prácticas corrosivas. 
Ejemplo de ello, es que sólo en EE.UU. la Comisión General de Comunicaciones registra anualmente decenas de miles de quejas de consumidores contra las empresas de telefonía celular. El patrón se comporta más o menos de la siguiente manera: Un carrier típico ofrece varias decenas de opciones de precios, que van desde los planes más económicos, con un número limitado de minutos, hasta planes muy costosos con garantías de miles de minutos. Cada plan tiene sus propios descuentos y restricciones. $Y$ aunque pueda parecer un modelo muy centrado en las necesidades del cliente (McGovern, 2007), la esencia de estos portafolios de servicios radica en la dificultad que tienen los usuarios para predecir su uso, castigándoselos por hablar demasiado tiempo o usado muy poco.

Si se analiza el comportamiento de la banca minorista, una estimación da cuenta que los consumidores estadounidenses pagaron sólo el 2007 más de U\$S 55.000 millones en comisiones por sobregiros. En este país las quejas de los consumidores fueron tan fuertes que la congresista por Nueva York, Carolyn Maloney logró reintroducir la Ley para Prácticas Limpias de Protección del Consumidor contra el Sobregiro, para evitar así el cobro abusivo de comisiones por parte de instituciones bancarias. Entonces, cuando emerge un nuevo actor en un nicho acotado de negocios, a partir de una propuesta distinta, se transforma no sólo en un competidor potente sino en un "agente de cambio".

Así lo demostró el banco online ING Direct, con una oferta amistosa para sus usuarios presentes y futuros, ofreciendo productos claros y fáciles de entender. Desde su lanzamiento, la entidad rechazó de plano la ortodoxia bancaria, ofreciendo cuentas de ahorro sin comisiones, sin tasas diferenciadas de interés y sin saldos mínimos, con cuentas corrientes simples, acceso sin recargo a una gran red de cajeros automáticos, un sitio web exento del caos de ofertas cruzadas, con un portafolio de ofertas catalogada como un ejemplo de simplicidad de productos y servicios. ¿Retornos obtenidos? Al 2007 el banco ING Direct era el cuarto banco de ahorro de EE.UU., con más de 100 mil clientes nuevos cada mes y actualmente con una base general de clientes cercana a los 22 millones de personas operando con más de 319.000 millones de euros transados en fondos de inversión.

Tácticas comerciales que a priori parecieran muy rentables, generan a largo plazo altas tasas de insatisfacción y descontento que pueden culminar en millonarias pérdidas a nivel de posiciones de mercado, y que repercuten con efecto halo produciendo manchas irreversibles y tremendamente corrosivas para la imagen e indicadores de reputación de una marca.

En consecuencia, asumiendo que en la impredecible vorágine del campo de las ciencias sociales no es recomendable proponer fórmulas taxativas o esquematizaciones reductivas, me atrevo a formular de manera prudente una ecuación simple que pudiera servir de complemento a las tradicionales fórmulas de valorización y estimación de dividendos reputacionales, basada en la casuística de algunos de los ejemplos organizacionales emblemáticos aquí mencionados, amparado bajo el principio de análisis Coetteris Paribus (congelamiento simulado de variables múltiples e incidentes):

\section{Ecuación: $\mathrm{EC}+\mathrm{MA}=>(\mathrm{VM})$ Rep}

C I I C : a , immmm $\mathrm{mmm}$ 
Ella explica la probabilidad del incremento reputacional de una organización, sobre la base de una construcción de identidad e imagen corporativa fundamentada y proporcional a sus acciones e intenciones (CIIC: a, i). Sobre esta plataforma, sumando una estrategia coherente (EC), más la implementación de técnicas y principios de marketing amigable (MA), es esperable una mayor valorización de marca (VM) e incremento del índice reputación asociado a la misma como factor multiplicador $>$ (VM) Rep

Esta ecuación simple pero adaptada a un campo de aplicación más bien social y comunicacional -al amparo de la ocurrencia de desviaciones o deltas posibles- también se reafirma a sí misma en la existencia de fórmulas que describen de manera muy pobre y parcial el problema de la valoración global de los intangibles, como el concepto de "capital intelectual", que para algunos autores se traduce a partir de la diferencia entre el valor de mercado ( > capitalización bursátil) y el valor en libros ( > valor contable), sin ofrecer explicaciones del por qué de esa distinta valoración, donde "capital intelectual" describe el problema pero lamentablemente no lo explica (Nieto, 2006).

Los tiempos que corren justamente demandarán un esfuerzo colectivo en este sentido, y aunque algunos pensadores se inclinen más por modelos orientados en mecanismos del control y fiscalización para no distraer la realidad empresarial (Reich, 2007), la reducción del riesgo parece ser una buena razón para terminar con las prácticas extractoras de valor, y hacerlo, representará a su vez, tanto para empresas y organizaciones a nivel mundial, la oportunidad de acceder a una diferenciación competitiva pero al mismo tiempo tangible.

El valor de las empresas se está supeditando cada vez más a la existencia, consolidación e incremento sostenido de activos intangibles, como la capacidad de aprendizaje de sus empleados y líderes o el talento para innovar en procesos y productos (Bitrán, 2009). Mientras el mundo se mueve abriéndose paso a través de una desordenada cascada de información económica, una nueva generación de líderes y expertos exploran nuevos nichos de negocios, especialmente en el rubro de la industria energética y la compra de miles de hectáreas para la emisión de bonos de carbono (CER's). Lo demuestra el caso en Chile de las deudas legadas por Forestal Trillium que hicieron que sus 270 mil hectáreas (que ocupan un tercio de la superficie de isla Tierra del Fuego-XII Región), fuesen adquiridas por Goldman Sachs, hoy transformadas en el emblemático proyecto Karukinka, catalogado como "El secreto verde mejor guardado", a través del cual exitosos hombres de negocios de la mano de científicos de renombre buscan demostrar que la sustentabilidad tiene dos apellidos y ejes: Ambiental y económico.

En el mapa de la Nueva Geografía Económica de Stiglitz y Volcker, el crecimiento económico comienza con la inversión, pero teniendo en cuenta que a los inversores lo que realmente les preocupa es la rentabilidad segura de su dinero, es determinante la valoración que tendrán estos paquetes accionarios en los mercados financieros presentes y futuros. Irrefutables datos empíricos, entregados por la hecatombe financiera en E.E.U.U. y el desplome bursátil por todos conocido, demuestran que la valoración en bolsa a nivel de negocios es cada vez más alejada de su valor contable, lo que permite afirmar que a nivel corporativo es irrenunciable considerar los activos intangibles como factor crítico de valorización, ya sea como política de marca, imagen corporativa, o grado de eficacia administrativa. Más importante aún, es referirse a la obligatoriedad de adoptar a nivel del cromosoma empresarial o institucional sólidos principios éticos, más no como acciones morales metamorfoseadas en acciones de marketing o mera comunica- 
ción de empresa, propios de sociedades postmoralistas (Lipovetsky, 2008), sino acciones o no acciones que permitan sumar real y duradero valor a su algoritmo reputacional.

Ha quedado en evidencia que son estos mismos mercados los que reaccionan sobre la base de "criterios extraeconómicos", a su vez influenciables por lo que Castell califica como "turbulencias de información" (2001), entre ellos, escenarios de incertidumbre política, transformaciones legales/judiciales, anticipaciones tecnológicas, desordenes a nivel de gobierno corporativo, incluso declaraciones o estados de ánimo provenientes de personalidades con capacidad de influencia sectorial. Tal vez se pueda responder la pregunta: ¿Por qué preocupa tanto la Reputación Corporativa? explicado parcialmente desde el proceso de estandarización de productos y servicios a nivel mundial (Montañés, 2006), si se considera que hoy para millones de nuevos y exigentes públicos es cada vez más difícil diferenciar éstos desde un enfoque meramente técnico. Es posible que, a partir de una consideración más cercana al marketing que inspirado en fundamentos meramente éticos, se pueda explicar las millonarias inversiones corporativas e incluso gubernamentales para esponsorizar y colaborar con tanto entusiasmo en loables proyectos de desarrollo sostenible, el fomento al deporte o la colaboración con causas justas, como medio y no fin, para transformar la reputación en beneficio de su propia imagen y capacidad de seducción para clientes, accionistas, usuarios o electores.

Uno de los más influyentes asesores de Barack Obama, en su análisis de la transformación de los mercados financieros globales, afirmó que "el flujo de los fondos y su valoración en los mercados financieros libres depende tanto de las percepciones como de la realidad objetiva", en otras palabras, en el entendido que en los tiempos que corren la percepción subjetiva se acopla cómodamente como parte de la realidad (Volcker, 2000).

Las manipulaciones financieras y las declaraciones destinadas a la creación de imagen, crean un ambiente informativo donde abunda la incertidumbre, y en donde los inversores se ven obligados a reaccionar en tiempo real, antes que la velocidad del mercado castigue cualquier vacilación. Y son estos millones de inversores individuales, incluso muchos de ellos con rostros de clientes (compra de promesa y ventas de expectativas) quienes al ser tan numerosos aumentan poderosa y abrumadoramente las fuentes de volatilidad para cualquier sector productivo, muchas veces sin ser capaces de responder a una lógica económica estricta, sino más bien bajo una lógica de complejidad caótica, resultado de la interacción entre millones de decisiones, percepciones y reacciones en tiempo real.

Sumado a toda esta complejidad y a la necesidad evidente de aunar esfuerzos, existe una percepción cada vez más extendida especialmente en la comunidad empresarial, sobre las ventajas asociadas a que una marca "sea parte de la solución en lugar de ser parte del problema".

Bajo este nuevo paradigma, y en una economía donde más de la mitad de los trabajadores se dedican a procesar información, tanto para organizaciones públicas o privadas, la tendencia es que las multitudes están prefiriendo por sobre las marcas registradas (MR) (trademarks) aquellas entendidas como trustmarks o marcas confiables, que en definitiva les otorguen garantías futuras, en términos de sus productos, servicios, decisiones o incluso para causas de apoyo, si fueren éstas auténticas, creíbles y por tanto confiables (Bricker, 2006), para convertirse en happymarks que marquen tendencia en comportamientos adquisitivos futuros.

En conclusión, más allá de la calidad del producto final, se busca la honradez del productor, el cumplimiento de una promesa, la garantía de apoyo post venta, la legitimidad de los procesos involucrados, se trate de una multinacional, corporación, institución de caridad o incluso la 
promesa de un líder político. Y en este proceso, cuanto más se democratiza la información, la demanda de opciones se hace prácticamente infinita. La mentalidad de los "sin alternativa quedó obsoleta, pues si hoy los ofrecimientos oficiales no bastan y se desploman, muchos emancipados y rupturistas ciudadanos crearán sus propias alternativas mediante sus propios canales para diseminar su legítima incomodidad.

El modelamiento de cada trustmarks como activo pro-valorización, se convertirá en una de las tareas sobresalientes para la alta gerencia, integrada como meta-objetivo y parte de la plataforma estratégica de cualquier división corporativa, para propiciar el desarrollo de comportamientos adquisitivos futuros sostenibles, a modo de una gran cuenta corriente y fondos de inversión a largo plazo, como elemento de intermediación con cada consumidor/ciudadano experto del siglo XXI y necesario para la construcción de puentes relacionales asísmicos.

\section{Referencias Bibliográficas}

Bitrán, E. (2009). Capital humano e innovación. Columna de opinión. La Tercera, (febrero 03). Sección Ideas \& debates.

Bricker, D. (2006). La reputación como algo perdurable. Todo gira alrededor de las marcas de confianza. Madrid: Ipsos Ideas.

Brynjolfsson, E.; Hitt, L. M. y Yang, S. (2000). Intangible Assets. How the interaction of computers and organization structure affects stock market valuations. Cambridge: MIT-Sloan School Center for e-business.

Cañibano, L., García-Ayuso, M.l y Sánchez, P. (2000). Accounting for intangibles: a literature review. Journal of Accounting Literature, vol. 19, pp. 102-130.

Castells, M. (2001). La galaxia Internet. Reflexiones sobre Internet, empresa y sociedad. Buenos Aires: Areté.

Corral, A.; Isusi, I.; Pérez, T.; San Miguel Unai. (2006). Contribución de las empresas a los objeti vos del milenio en Latinoamérica. Equipo de trabajo de IKEI Research \& Consultancy y Banco Interamericano de Desarrollo (Sub departamento de empresa privada y mercados financieros).

Covey, S. M. R. (2008). El factor confianza. El valor que lo cambia todo. Buenos Aires: Ediciones Paidós Ibérica S.A.

David. A. Aaker. (1992). Managing Brand Equity. Paper: The Value of Brand Equity. Haas School of Business. University of California at Berkeley.

Dev, C. S. (2008). La marca corporativa: ¿Ayuda u obstáculo. Harvard Business Review. Volumen 86. $\mathrm{N}^{\circ} 2$.

Eccles, R. G., Newquist, S. y Schatz, R. (2007). La reputación y sus riesgos. Harvard Business Review. Volumen $85 . \mathrm{N}^{\circ} 2$.

Fernández L., J. (2002). Gestionar la confianza. Un modelo integrador de las políticas de marketing y gestión de personas para alcanzar la excelencia. Madrid: Financial Times-Prentice Hall.

Fernández, P. (2005). Guía rápida de valorización de empresas. Madrid: Ediciones Gestión 2000.

Laszlo, C. (2008). Sustainable value. How the world's leading companies are doing well doing good. Standford, California: Stanford business books.

Lipovetsky, G. (2008). El crepúsculo del deber. La ética indolora de los nuevos tiempos democráticos. Barcelona: Anagrama

M.Schultz, R. (2009). Seis formas en que las empresas gestionen mal el riesgo. Volumen 87 número 3. 
Marchesán, A. (2005). Comunicación productiva en la era de las relaciones. Buenos Aires: Gran Aldea Editores.

Maturana, H. (1999). Transformación en la convivencia. Santiago: Dolmen Ediciones S.A.

McGovern, G. y Moon, Y. (2007). Las empresas y los clientes que las odian. Harvard Business Review. Volumen $85 \mathrm{~N}^{\circ} 6$.

Melnick, S. I. y Barraza, J.(2008). E.T.A.N. Estrategia, tecnología, adaptabilidad y negocios. Diseño e implementación de estrategias exitosas de negocios para el siglo 21. Santiago: Anticipa S.A. y Escuela de Negocios Universidad Adolfo Ibáñez.

Montañés, P. y Olier, E. (2006). Corporate governance intelligence. Desarrollando la corporación en la web. Madrid: Prentice Hall Financial Times.

Nieto, J. (2006). Estructura, Estrategia y Conocimiento. Una lectura histórica de la política de gestión (Tesis Doctoral). España: ESADE

Pullen, W. y Godoy, S. (2008). Estudio de Reputación Corporativa. Reputation Institute y Pontificia Universidad Católica de Chile, Facultad de Comunicaciones.

Reich, R. (2007). Supercapitalism: The transformation of Business, democracy, and everyday life. New York: Borzoi Books.

Sorman, G. (2008). La Economía no miente. Falta ciudad: Editorial Sudamericana.

The McKinsey Quarterly (2008). The state of corporate philantrophy. Miami: McKinsey Global Survey.

The Strategic Planning Institute, (s/f). Profit Impact of Market Strategy. Disponible en: http://pimsonline.com/index.htm

Villafañe, J. (2006). La comunicación empresarial y la gestión de los intangibles en España y Latinoamérica. Estudio anual 2006. Departamento de Comunicación Audiovisual y Publicidad, Universidad Complutense de Madrid. Madrid: Pearson Prentice Hall.

Volcker, P. (2000). A sea of global finance. En Will Hutton y Anthony Giddens (ed.), On the Edge. Living in Global Capitalism. Londres: Jonathan Cape

Yates, C. (2008). La empresa sabia. Madrid: Editorial Díaz Santos.

Summary: The following article approaches corporate reputation, relating it to the brand value and tying it to the management of assets and liabilities, in the frame of a communicational platform. In the article a reflection is made around the process of planning, maintenance and control of assets linked to reputation, in which Public Relations are involved as well as other disciplines. In addition, sensitivity of the organization bonds with its stakeholders is deeply analyzed. For this, it approaches consequences of reputation risk and the concept of transforming organizations, in a permanent monitoring of the intangible resources on communication and management platforms, to allow that their logos could be able to tell a corporate history, with relevance to strategic targets and integrity for his credibility.

Key Words: brand - communications people - Corporate reputation - institutional communication organizational communication - public - Public Relations - stakeholders - strategic communication.

Resumo: Este artigo aborda a temática da reputação corporativa, relacionando-a ao valor de marca e vinculando-a à gestão do conjunto de ativos e passivos, sobre uma plataforma 
de comunicação. Para isto, reflexiona em torno do processo de planejamento, manutenção e controle do ativo da reputação, nos que têm ingerência -entre outros- os esforços das Relações Públicas, analisando ao mesmo tempo a sensibilidade da relação da organização com os públicos estratégicos. Para isto, aborda as implicancias do risco da reputação e a concepção das organizações de transformação, numa vigilância permanente dos recursos intangíveis sobre plataformas de comunicações e de gestão, para conseguir que em verdade os seus logos sejam capazes de contar uma história corporativa, com pertinência a objetivos estratégicos e integridade para sua credibilidade.

Palavras chave: comunicação estratégica - comunicação institucional- comunicação organizacional - Comunicadores - marcas - públicos - Relações Públicas - Reputação corporativa - stakeholders.

${ }^{*}$ Magíster en Comunicación Estratégica de la Universidad Adolfo Ibáñez. Diplomada en Gestión de Responsabilidad Social de la Pontificia Universidad Católica de Valparaíso y Sofofa, Santiago, Chile. [Ver CV completo en la página 151] 